\title{
Effects of affinity on binding of HER2-targeting Affibody molecules: Model experiments in breast cancer spheroids
}

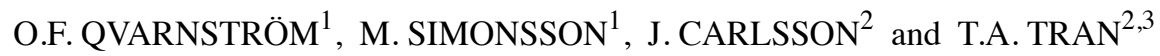 \\ Sections of ${ }^{1}$ Oncology and ${ }^{2}$ Biomedical Radiation Sciences, Department of Radiology, \\ Oncology and Radiation Sciences, Rudbeck Laboratory, Uppsala University, SE-751 85 Uppsala; \\ ${ }^{3}$ Lund University Bioimaging Center, Lund University, SE-221 84 Lund, Sweden
}

Received February 23, 2011; Accepted April 2, 2011

DOI: $10.3892 /$ ijo.2011.1045

\begin{abstract}
Binding of a targeting agent in tumor tissue is influenced by many factors such as molecular weight, charge and affinity of the targeting agent and vascularization of the tumor. In this study, we analyzed tumor cell binding of three HER2-specific and radiolabeled Affibody molecules with different affinities. The Affibody molecules had affinities in the range of 0.12-3.8 $\mathrm{nM}$. Cellular binding was analyzed, after $2 \mathrm{~h}$ of incubation, in tumor spheroids composed of BT474 breast cancer cells, which highly express HER2. Binding was, due to the binding-site barrier, limited to the outer $15 \pm 5 \mu \mathrm{m}$ rim of the spheroids, independent of affinity when the concentration of the substances was low. When the concentration was high, the binding site barrier was overcome and the binding occurred approximately $35 \pm 5 \mu \mathrm{m}$ into the spheroids for the two high affinity substances and $50 \pm 5 \mu \mathrm{m}$ for the low affinity substance. The lower affinity might allow for penetration into deeper regions due to less firm binding. We conclude that there is a binding site barrier within tumor spheroids which can be overcome by increased concentration of substance and modified by affinity.
\end{abstract}

\section{Introduction}

The depth of binding of tumor targeting agents in non-vascularized micro-metastases can be studied using the spheroid in vitro model $(1,2)$. The advantage of the spheroid model is that it allows the study of penetration, binding and action of diagnostic and therapy-effective substances under strictly controlled conditions. Animal models with transplanted tumors are instead of value when variations in kidney clearance,

Correspondence to: Dr Thuy A. Tran, Unit of Biomedical Radiation Sciences, Department of Radiology, Oncology and Radiation Sciences, Rudbeck Laboratory, Uppsala University, SE-751 85 Uppsala, Sweden E-mail: thuy.tran@med.lu.se

Key words: Affibody, affinity, binding, breast cancer cells, BT474, cell spheroids, compound development, HER2, penetration, radionuclides, binding-site barrier vascularization, nutrition supply, host defense and other factors are allowed to influence the depth of binding $(3,4)$.

The spheroids share several structural features with tumor nodules, especially small non-vascularized micro-metastases. The spheroids are spherically shaped with an outer proliferative cell layer, an inner cell layer of mainly quiescent cells and a central core consisting of necrotic cells $(2,5-8)$. The central area of necrosis most often contains an amorphous degenerative mass or a more or less homogeneous assembly of pycnotic cells. Apoptosis can be seen in the surrounding cell layers and the extent of apoptosis is dependent on the therapeutical strategy employed (9-12). The spheroids have radial gradients in terms of $\mathrm{pH}, \mathrm{pO}_{2}$, nutrition supply and catabolic products, similar to those found in metastatic tumor nodules in vivo $(2,13)$.

A number of factors influence the efficacy of a tumor targeting agent, such as the cellular distribution of the targeted antigen and the interaction between the antibody and the antigen. This interaction is influenced by the binding specificity, binding affinity, cellular internalization and catabolism of the targeting agent. Thus, binding affinity is one of the most important parameters which should be considered in the design of tumor targeting agents. Weinstein and van Osdol (14) have described a relationship between affinity and penetration, the so-called binding-site barrier, meaning that increased affinity decreases the penetration of a targeting agent due to the strong interaction with the antigen and thereby prevents movement of the binding substance deeper into the tumor mass. The factors predicted to affect the extent of the binding-site barrier are antigen density, internalization and metabolism, and binding affinity $(3,14,15)$.

The binding of three ${ }^{125}$ I-labeled Affibody molecules, with different affinities, to the tumor associated receptor HER2 was analyzed in cellular spheroids in this study. HER2 is overexpressed in several cancers, especially breast cancer and is an interesting target for both diagnostic and therapeutical purposes (16-18).

Affibody molecules have previously been produced yielding affinities in the mid to low nanomolar range, against many different target proteins, including HER2 (19), transferrin (20), amyloid $\beta$ peptide (21), EGFR (22), Factor VIII (23), CD25 (24), HIV gp120 (25), and CD28 (26). Affibody molecules belong to the category of scaffold proteins, in which a binding 
interface is engineered in the so-called Z-domain (27). The Affibody molecule library was constructed by combinatorial randomization of 13 solvent accessible residues. The Affibody libraries have been displayed using phage display for selection of the best binders. Efforts to increase affinity have been successful in several cases $(23,28-30)$, where affinity maturations have been achieved either by helix shuffling (29) or sequence alignment and directed combinatorial mutagenesis $(23,28,30)$.

The Affibody molecules have been described to have a number of attractive properties useful in a variety of settings, such as biotechnological applications and potentially for therapy and molecular imaging (31-33). They have a small size $(\sim 7 \mathrm{kDa})$, are highly soluble and stable and are small enough for solid-phase peptide synthesis, hence facilitating the introduction of desired fluorophores or modifications for radiolabeling. Furthermore, the lack of cysteines makes the Affibody molecules suitable for site-specific labeling (27).

\section{Materials and methods}

Synthesis and biochemical analysis of Affibody molecules. Three HER2-specific Affibody molecules, denoted as AB1, AB2 and AB3, were chemically synthesized. The amino acid sequence of these Affibody molecules was obtained from the Affibody library (30). The backbones of the Affibody molecules are identical for all molecules, with the exception of some amino acids at the randomized positions making up the antigen-binding region. The different amino acids at these positions confer different binding affinities to the target protein. These amino acid sequences were somewhat modified to be suitable for assembly with the Fmoc-strategy on a fully automated peptide synthesizer and purified and characterized as described earlier (34). Another Affibody molecule, specific for the human amyloid beta $(\mathrm{A} \beta)$ peptides in Alzheimer disease (21) and with no binding to HER2, was used as control.

A Biacore ${ }^{\circledR} 2000$ instrument (Biacore AB, Uppsala, Sweden) was used for real-time biospecific interaction analysis (BIA) between the synthetic Affibody molecules and HER2 protein as described earlier (34). This was done to determine on-rates, off-rates and affinity of the Affibody molecules to HER2. Briefly, the extracellular domain of HER2 (Pierce, Rockford, IL, USA) was immobilized on a CM5 sensor chip by amine coupling. HER2 was diluted to $10 \mu \mathrm{g} / \mathrm{ml}$ in $20 \mathrm{mM} \mathrm{NaOAc}$, $\mathrm{pH} 4.5$, and the immobilization levels reached 1800 response units (RU). The different Affibody molecules were diluted to various concentrations in HBS-EP buffer (0.01 M HEPES, $\mathrm{pH} 7.4,0.15 \mathrm{M} \mathrm{NaCl}, 3 \mathrm{mM}$ EDTA, $0.005 \%$ surfactant P20), all from Biacore $\mathrm{AB}$. The binding kinetics were studied in a 5-min association phase and a 30-min dissociation phase with a flow rate of $50 \mu \mathrm{l} / \mathrm{min}$, followed by regeneration with $25 \mathrm{mM}$ $\mathrm{HCl}$. Kinetic constants were estimated using the BIAEvaluation 4.1 software (Biacore $\mathrm{AB}$ ), assuming one-to-one binding.

Radiolabeling with ${ }^{125} \mathrm{I}$. Affibody molecules (10 $\mu 1,1 \mathrm{mg} / \mathrm{ml}$ in PBS) were mixed with ${ }^{125} \mathrm{I}$ as $\mathrm{NaI}$ solution (4 MBq) using the Chloramine-T (CAT, Sigma St. Louis, MO, USA) method previously described (35). The reaction was initiated by adding CAT in PBS $(10 \mu l, 2 \mathrm{mg} / \mathrm{ml})$ and incubated for $1 \mathrm{~min}$ during vigorous shaking and then terminated by adding sodium metabisulfite $(20 \mu \mathrm{l}, 2 \mathrm{mg} / \mathrm{ml})$. Labeled Affibody molecules were separated from non-reacted ${ }^{125}$ I and low-molecular-weight reaction components by size-exclusion chromatography on a NAP-5 column (Sephadex G-25, GE Healthcare) pre-equilibrated with PBS. The specific activity was $\sim 0.32 \mathrm{MBq} / \mu \mathrm{g}$.

Cell culture. The breast cancer BT474 cells (American Type Culture Collection, Manassas, VA, USA) were grown as both monolayer cultures and cell spheroids in Ham's F10 cell culture medium (Biochrome KG, Berlin, Germany) as previously described by Qvarnström et al (12). All culture media were supplemented with $10 \%$ fetal bovine serum, L-glutamine (2 mM) and PEST (100 IU/ml penicillin, $100 \mu \mathrm{g} / \mathrm{ml}$ streptomycin), all from Biochrome KG (Berlin, Germany). We describe this culture medium as 'complete medium' throughout the rest of the text. The cells were cultured at $37^{\circ} \mathrm{C}$ in an incubator with humidified environment with $5 \% \mathrm{CO}_{2}$. The cells were trypsinized with trypsin-EDTA $(0.25 \%$ trypsin, $0.02 \%$ EDTA in phosphate-buffered saline (PBS) without $\mathrm{Ca}$ and $\mathrm{Mg}$ ), from Biochrome $\mathrm{KG}$.

Binding specificity studies on monolayers. ${ }^{125}$ I-labeled Affibody molecules, AB1-AB3, were added to two groups of Petri dishes (three dishes per group) with a calculated ratio of one radiolabeled Affibody molecule per HER2 receptor. One group of dishes was pre-saturated with a 100-fold excess of non-labeled Affibody molecules $10 \mathrm{~min}$ before the labeled molecules were added. Cells were incubated for $1 \mathrm{~h}$ at $37^{\circ} \mathrm{C}$, and the incubation medium was then collected. The cell dishes were washed six times with cold serum-free medium and treated with $0.5 \mathrm{ml}$ trypsinEDTA for $10 \mathrm{~min}$ at $37^{\circ} \mathrm{C}$. When the cells were detached, $0.5 \mathrm{ml}$ complete medium was added to each dish and the cells were resuspended. The cell suspension was used for radioactivity measurements.

Growth of spheroids. Monolayer cultures of BT474 cells were trypsinized for $10 \mathrm{~min}$ at $37^{\circ} \mathrm{C}$ and the cell suspension was diluted in complete culture medium and seeded in spinner flasks. The spinner flasks were allowed to rotate around a horizontal axis, with a speed of about $50 \mathrm{rpm}$, and contained $125 \mathrm{ml}$ complete culture medium. Hundreds of spheroids formed spontaneously and started to grow. All spinner cultures were grown at $37^{\circ} \mathrm{C}$ in an incubator in a humidified environment at $5 \% \mathrm{CO}_{2}$. The normal growth rate of the spheroids was evaluated as described earlier (12). The diameter of the spheroids was measured using an ocular scale, in the eyepiece of an inverted microscope. Their volume was calculated with the relation $V=4 / 3 \times \pi \times(a \times b)^{3 / 2}$ where $a$ and $b$ were radii measured at right angles by means of an ocular scale, in the eyepiece of an inverted microscope (Leica DMIL, Göteborg, Sweden).

Binding studies in spheroids. Four BT474 spheroids per group were used for binding studies. Spheroids were transferred and incubated in roller flasks containing a 3-ml solution of labeled Affibody molecules, either at a concentration of 100 times excess of protein to HER2 receptors $(\sim 107 \mathrm{kBq} / \mathrm{ml})$, or 500 times excess of protein to HER2 receptors $(\sim 534 \mathrm{kBq} / \mathrm{ml})$. The roller flasks were rotated to maintain a mixing during the whole treatment of $2 \mathrm{~h}$ at $37^{\circ} \mathrm{C}$ in an incubator with humidified environment with $5 \% \mathrm{CO}_{2}$. 
Fixation and sectioning. Spheroids were, after the incubation washed in complete medium, fixed in formalin for $20 \mathrm{~h}$, dehydrated in a graded alcohol scale (from 70 to $100 \%$ ), infiltrated by xylene and paraffin using a VIP (Vacuum infiltrating processor, Ventana Renaissance, Ventana Medical Systems, Tucson, AZ, USA), embedded in paraffin blocks and sectioned to a thickness of about $5 \mu \mathrm{m}$ per section. Central sections, from the maximal diameter of the spheroids, were selected for analysis. These sections were de-paraffinized in xylene, washed in alcohol, and processed further for autoradiography as previously described (12). The dipping technique using K5 emulsion (Ilford Scientific, London, UK) was applied and the samples were then left in the dark for a month and then developed in Kodak D19 and fixed with UNIFIX (Kodak, Rochester, USA). Some sections were stained with hematoxylin.

${ }^{3} \mathrm{H}$-TdR-labeling. Experiments with ${ }^{3} \mathrm{H}-\mathrm{TdR}$ labeling of the BT474 spheroids have been reported previously by our research group (12) and the procedures are only briefly described here. BT474 spheroids were incubated with ${ }^{3} \mathrm{H}-\mathrm{TdR}$ (specific radioactivity of $5 \mathrm{Ci} / \mathrm{mmol}$, Radiochemical Centre, Amersham, London, UK) in culture medium with $37 \mathrm{kBq} / \mathrm{ml}$ for $24 \mathrm{~h}$ in spinner flasks. The spheroids were then washed in complete medium and fixed, embedded in paraffin and sectioned. The sections were processed for autoradiography as described above.

Immunohistochemistry. Immunohistochemical staining was performed in a Benchmark IHC/ISH (Ventana Medical Systems, Tucson, AZ) using a monoclonal antibody against HER2 (antibody code A0485, Dako Sweden AB, Stockholm, Sweden). Staining was enzymatically developed with 3, 3-diaminobenzidine-tetra hydrochloride [DAB, Dako Sweden AB. Such procedures have been described in more detail $(36,37)]$.

Digital image analysis. Images of Affibody-stained spheroids were acquired with a Spot Insight CCD-camera (Diagnostic Instruments, Sterling Heights, Michigan) connected to a Nikon Eclipse E400 microscope (Nikon Corp., Tokyo, Japan). Images were acquired at magnification $\mathrm{x} 40$. The microscope light source and camera was calibrated before all image acquisition sessions to ensure reproducible light conditions and white balance levels. The stained area was determined by a series of digital image analysis algorithms implemented in $\mathrm{Java}^{\mathrm{TM}}$ and the Java Advanced Imaging (JAI) platform. The labeled structures in the images were extracted by binary segmentation using a fixed gray level threshold (38). The software was designed so that $10 \times 30 \mu$ m-regions were defined along radial drawn lines from the surface of the spheroids towards the center, so the selected regions were aligned perpendicular to the surface (Fig. 2). Labeled areas and total areas of the selected regions were simultaneously recorded for each region. This provided a measure of staining density areas from the surface to depths of $100 \mu \mathrm{m}$. A total of 84 spheroid sections were analyzed.

\section{Results}

Binding affinities determined by Biacore analysis. The binding kinetics of the three Affibody molecules AB1-AB3 is shown
Table I. Binding kinetics of the three Affibody molecules, AB1-AB3. ${ }^{\mathrm{a}}$

\begin{tabular}{lrcr}
\hline & $\mathrm{k}_{\mathrm{a}}(1 / \mathrm{Ms})$ & $\mathrm{k}_{\mathrm{D}}(1 / \mathrm{s})$ & $\mathrm{K}_{\mathrm{D}}(\mathrm{nM})$ \\
\hline AB1 & $(6.3 \pm 1.1) \times 10^{6}$ & $(7.37 \pm 1.33) \times 10^{-4}$ & $0.12 \pm 0.03$ \\
AB2 & $(9.2 \pm 1.8) \times 10^{6}$ & $(1.43 \pm 0.25) \times 10^{-3}$ & $0.16 \pm 0.04$ \\
AB3 & $(6.69 \pm 0.01) \times 10^{6}$ & $(2.55 \pm 0.12) \times 10^{-2}$ & $3.81 \pm 0.18$
\end{tabular}

${ }^{a}$ Results from Biacore analysis. $\mathrm{k}_{\mathrm{a}}$ is the association rate (on-rate), $\mathrm{k}_{\mathrm{d}}$ is the dissociation rate (off-rate) and $K_{D}=k_{d} / k_{a}$ is the affinity or binding strength at equilibrium. Note that high affinity corresponds to small $\mathrm{K}_{\mathrm{D}}$ values.

Table II. Binding specificity of the three radiolabeled Affibody molecules: ${ }^{125} \mathrm{I}-\mathrm{AB} 1,{ }^{125} \mathrm{I}-\mathrm{AB} 2$ and ${ }^{125} \mathrm{I}-\mathrm{AB} 3$ to monolayer cultured BT474 cells. ${ }^{\mathrm{a}}$

\begin{tabular}{llcr}
\hline & \multicolumn{2}{c}{ Cell-associated radioactivity of added, $\%$} \\
\cline { 2 - 4 } & Non-blocked & Blocked & P-value \\
\hline${ }^{125} \mathrm{I}-\mathrm{AB} 1$ & $25.1 \pm 1.8$ & $1.0 \pm 0.2$ & $1.9 \times 10^{-5}$ \\
${ }^{125} \mathrm{I}-\mathrm{AB} 2$ & $31.7 \pm 0.61$ & $0.64 \pm 0.03$ & $9.9 \times 10^{-8}$ \\
${ }^{125} \mathrm{I}-\mathrm{AB} 3$ & $32.9 \pm 0.45$ & $0.49 \pm 0.14$ & $2.9 \times 10^{-8}$ \\
\hline
\end{tabular}

${ }^{a}$ Mean values and standard deviations from 3 dishes. P-values assessed by Student's t-test. When an excess of non-radioactive Affibody molecules were added in advance (blocked), the binding of the radioactive corresponding molecules decreased significantly proving specific binding.

in Table I. AB1 and AB2 had high and rather similar affinities $\left(\mathrm{K}_{\mathrm{D}}\right), 0.12$ and $0.16 \mathrm{nM}$, respectively, while the affinity of AB3 was lower; $3.8 \mathrm{nM}$. Note that low values mean high affinity and higher values mean lower affinity.

Binding specificity. The obtained binding specificities are shown in Table II. All three Affibody molecules had specific binding to HER2 since the signal could be blocked to nearly two orders of magnitude by an excess of non-labeled substance.

Growth of breast cancer BT474 spheroids. The growth rate of the spheroids was high during the first three days and thereafter decreased and then reached a plateau after a week, as previously reported (12). The spheroids taken for experiments had diameters in the range $700-850 \mu \mathrm{m}$ and volumes in the range (170-300) $\mathrm{x} 10^{6} \mu \mathrm{m}^{3}$. The volume doubling time was, for these spheroids, about 3 days. The proliferation gradient, measured by counting the number of ${ }^{3} \mathrm{H}-\mathrm{TdR}$ labeled cell nuclei per area, decreased drastically as a function of distance, from about $60 \%$ at the surface down to a just a few percent near the necrotic area, i.e. about $180 \mu \mathrm{m}$ inside the spheroids. The cell density seemed constant in the outer cell layers of the spheroids and decreased in the deeper regions near the necrosis, and such data have been published (12). 

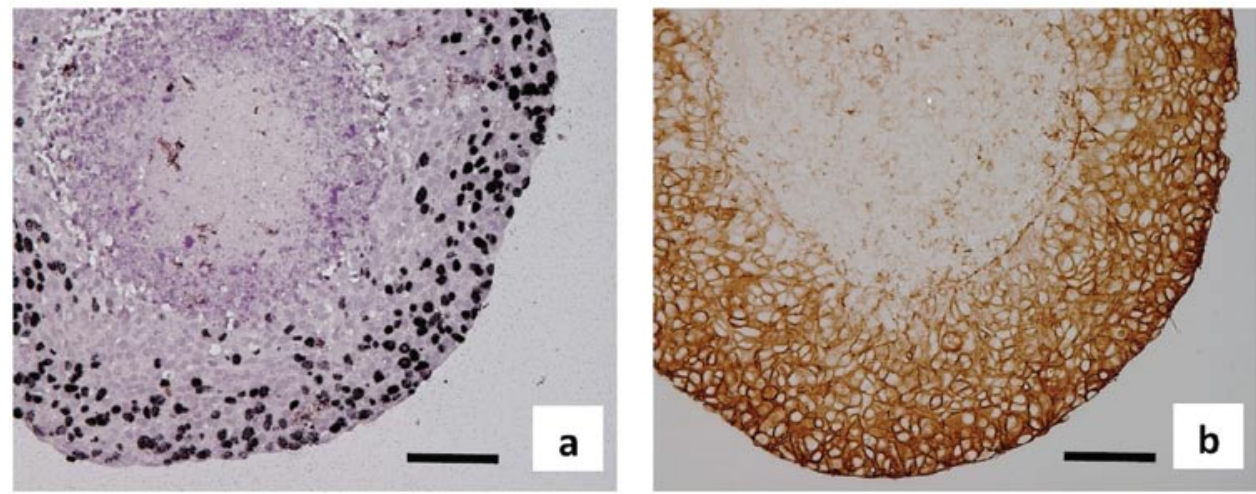

Figure 1. Morphological appearance of a BT474 spheroid (diameter, $800 \mu \mathrm{m}$ and sectioned in $5 \mu \mathrm{m}$ slices). (a) Spheroid processed for autoradiography after 24-h incubation with ${ }^{3} \mathrm{H}-\mathrm{TdR}$. The dark spots in the section show heavily labeled cell nuclei, indicating that these cells were, or have been, in the DNA synthesis phase (S-phase) during the incubation period. The blue color is haematoxylin staining. (b) Spheroid section stained for HER2 expression. Note the strong membranous staining in the viable cell layer and the somewhat weaker staining near the necrosis. The central necrosis is an amorphous mass in the centre of the spheroids and contained neither S-phase cells nor HER2 staining. The bars indicate $100 \mu \mathrm{m}$.

a

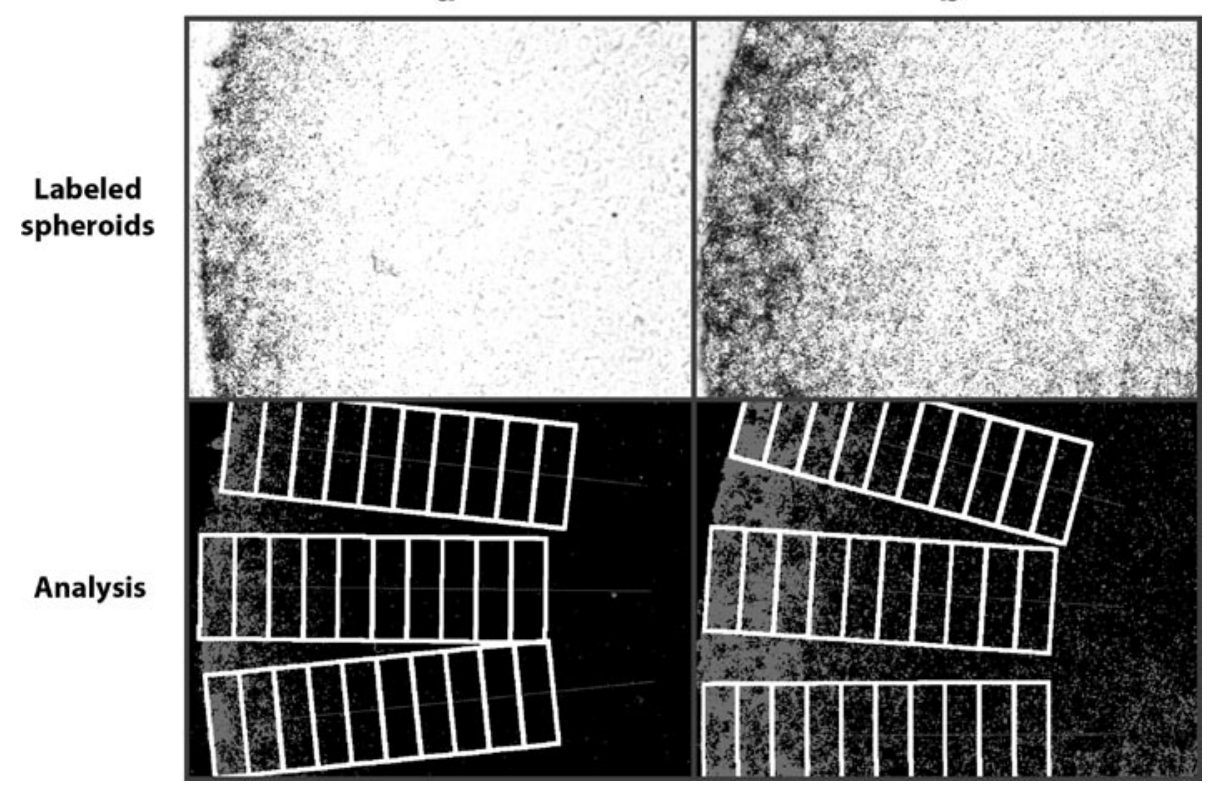

Figure 2. Analysis of autoradiography sections from spheroids incubated with ${ }^{125} \mathrm{I}-\mathrm{AB} 2$ at two different concentrations (a) $100 \mathrm{x}$ and (b) 500x where $100 \mathrm{x}$ and 500x means 100 and 500 times more Affibody molecules than the calculated number of HER 2 receptors. Regions of $10 x 30 \mu \mathrm{m}$ were defined by the software, along radial lines starting from the surface and directed toward the centre of the spheroids.

Morphological appearance of BT474 spheroids. The typical morphological appearance of BT474 spheroids is shown in Fig. 1. There were only ${ }^{3} \mathrm{H}-\mathrm{TdR}$ labeled cells in the outer peripheral regions. HER2 staining is also shown and the membranous staining is strong in the viable cell layer, although somewhat weaker near the border to necrosis.

Analysis of Affibody molecule binding in spheroids. Sections of spheroids, incubated with ${ }^{125} \mathrm{I}-\mathrm{AB} 2$, illustrate the analysis procedure, see Fig. 2. Regions of 10x30 $\mu \mathrm{m}$ were defined by the software, along radial lines starting from the surface and directed to the centre of the spheroids. The areas were selected by a fixed binary threshold level and measured separately for each region. Background levels were analyzed and subtracted. Note that 500x excess of substances, in relation to the amount of receptors, resulted in binding deeper inside the spheroids in comparison to the $100 \mathrm{x}$ excess. The unspecific radiolabeled control Affibody molecule, binding human amyloid beta $(\mathrm{A} \beta)$ peptides in Alzheimer disease, did not give detectable autoradiographic tracks and therefore served as a good negative control.

Calculated binding depths. Binding curves, normalized to unity at the periphery, and obtained from the image analysis of binding patterns are shown in Fig. 3. Binding depths were calculated as the distance from the periphery at which the grain density decreased to $50 \%$ of the maximal value at the periphery. The binding depths were only $15 \pm 5 \mu \mathrm{m}$ for all three Affibody molecules when they were given at low concentration (100x, Fig. 3a) and this was probably due to the binding-site barrier. When the concentration increased (500x, Fig. 3b) the binding-site barrier was overcome and the binding depth was 
a

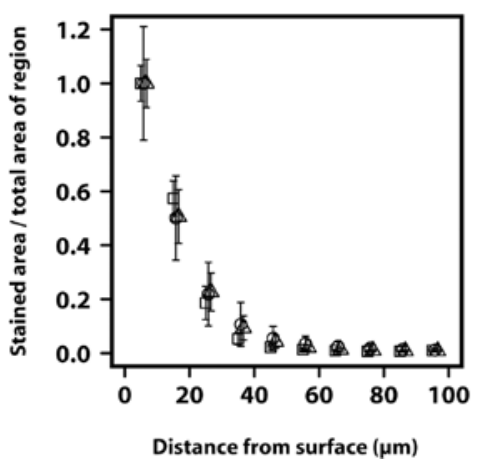

b

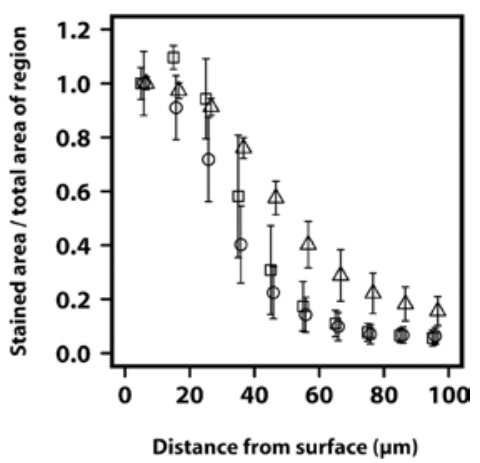

Figure 3. Quantitative evaluations of binding of ${ }^{125} \mathrm{I}-\mathrm{AB} 1$ (squares), ${ }^{125} \mathrm{I}-\mathrm{AB} 2$ (circles) and ${ }^{125} \mathrm{I}-\mathrm{AB} 3$ (triangles) as a function of distance from the surface in the spheroids. All spheroids were incubated with (a) the low concentration (100x) and (b) with the high concentration (500x). All data were normalized to unity in the most peripheral region. Mean values and standard deviations are shown.

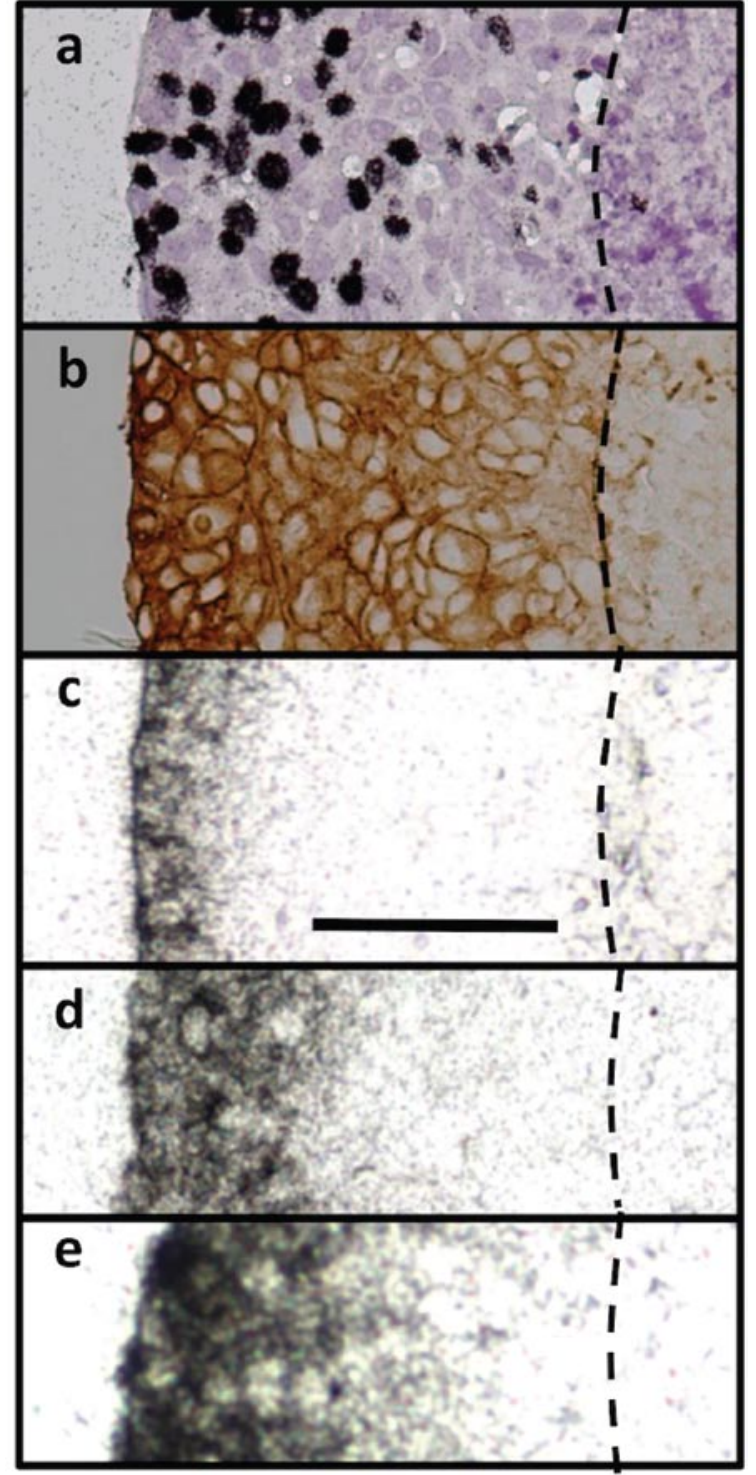

Figure 4. Properties of, and penetration in, the viable cell layers of the spheroids. (a) ${ }^{3} \mathrm{H}-\mathrm{TdR}$ labeling and haematoxylin staining, (b) HER2 staining, (c) binding of ${ }^{125} \mathrm{I}-\mathrm{AB} 1$ (low concentration), (d) binding of ${ }^{125} \mathrm{I}-\mathrm{AB} 1$ (high concentration), (e) binding of ${ }^{125} \mathrm{I}-\mathrm{AB} 3$ (high concentration). The bar in (c) marks $100 \mu \mathrm{m}$ and this is valid for all cases. The dashed lines indicate the border to necrosis (positioned to the right of the dashed lines). then $35 \pm 5 \mu \mathrm{m}$ for ${ }^{125} \mathrm{I}-\mathrm{AB} 1$ and ${ }^{125} \mathrm{I}-\mathrm{AB} 2$, and $50 \pm 5 \mu \mathrm{m}$ for ${ }^{125} \mathrm{I}-\mathrm{AB} 3$. The higher value for ${ }^{125} \mathrm{I}-\mathrm{AB} 3$ was probably due to the lower binding affinity allowing greater penetration to the deeper regions.

Properties of, and penetration into, the viable cell layers. Fig. 4 summarizes the results regarding biology and Affibody molecule binding in the BT474 spheroids; a) ${ }^{3} \mathrm{H}-\mathrm{TdR}$ labeling, b) HER2 staining, c) ${ }^{125} \mathrm{I}-\mathrm{AB} 1$ binding (low concentration), d) ${ }^{125} \mathrm{I}-\mathrm{AB} 1$ binding (high concentration) and e) ${ }^{125} \mathrm{I}-\mathrm{AB} 3$ binding (high concentration). The dashed lines indicate the position of necrosis. The selected spheroids had a diameter of about $850 \mu \mathrm{m}$ and the viable cell layers were $\sim 80 \mu \mathrm{m}$ in these cases. The images are, for ${ }^{3} \mathrm{H}-\mathrm{TdR}$ labeling and HER2 staining, good illustrations of their biological properties. High ${ }^{3} \mathrm{H}-\mathrm{TdR}$ labeling was only evident in the periphery while HER 2 staining was apparent all the way into the necrotic area.

The binding of the Affibody molecules was mainly in the periphery, while HER2 was also expressed in the deeper regions. Increased concentration of ${ }^{125} \mathrm{I}-\mathrm{AB} 1$, from $100 \mathrm{x}$ to $500 \mathrm{x}$, gave binding in the deeper regions, probably due to saturation of HER2 binding sites in the periphery so that the binding site barrier was overcome. The lower affinity of ${ }^{125} \mathrm{I}-\mathrm{AB} 3$ gave, at high concentrations, even better penetration than the high affinity molecule ${ }^{125} \mathrm{I}-\mathrm{AB} 1$ although they were given at the same high concentration and this was probably due to less firm binding (lower affinity) allowing better penetration. Binding of ${ }^{125} \mathrm{I}-\mathrm{AB} 2$ is not shown in Fig. 4 since the binding patterns were similar to those of ${ }^{125} \mathrm{I}-\mathrm{AB} 1$, which was expected since the difference in affinity was small between these Affibody molecules. Examples of ${ }^{125} \mathrm{I}-\mathrm{AB} 2$ binding are instead shown in Fig. 3.

\section{Discussion}

Our findings primarily illustrate that the binding-site barrier limits the binding of Affibody molecules to the outer thin cell layer of the spheroids, independent of affinity, when the concentration of the substances was low. However, at higher Affibody concentrations, the binding-site barrier seemed to be overcome and the binding occurred into the deeper regions 
of the spheroids. Furthermore, at the high concentrations, the Affibody molecules with the lower affinity bound deeper inside the spheroids than those with high affinity. The reason for this may be that a less firm binding allowed the molecules to bind and detach repeatedly at a high frequency and thereby giving them the capacity to move deeper into the spheroids.

The HER2 binding Affibody molecules had affinities in the range of 0.12-3.8 $\mathrm{nM}$ and it has, in previous reports $(3,15)$, been indicated that an affinity of about $1 \mathrm{nM}$ might be optimal for maximal uptake. The study by Adams et al (3) was performed with transplanted tumors in nude mice and further research is necessary since many factors have to be considered in vivo, e.g., penetration through the tumor capillary walls and renal clearance.

All three radiolabeled Affibody molecules had specific binding to HER2 since the binding could be blocked nearly two orders of magnitude by an excess of non-labeled corresponding substance. The control substance, i.e., the radiolabeled Affibody molecule specific for the human amyloid beta $(A \beta)$ peptides in Alzheimers disease, showed no measurable binding at any depth of the spheroids and therefore served well as a non-specific control.

Thus, binding affinity of a molecule is evidently an important parameter which should be considered in the design of a tumor-targeting agent. Weinstein et al (14) described the binding-site barrier as a phenomenon where increased affinity of a substance decreases the penetration due to strong interaction with the antigen. This is similar to that observed in affinity chromatography where the substances get stuck at the first part of the column and the binding substances cannot pass. The reason for this is, also in this case, that there is an abundance of binding sites in relation to the amount of substances trying to pass, so that the substances essentially become trapped. Factors predicted to affect the extent of the binding-site barrier in cellular systems are; antigen density, binding affinity, internalization and metabolism of the targeting agent $(3,14,15)$. For further experiments it could be useful to produce molecules with both lower and higher affinity than those applied in this study. Affibody molecules have previously been produced yielding affinities in the mid to low nanomolar range, against many different target proteins as described in the introduction. Efforts to increase Affibody affinity with affinity maturation procedures, have also been successful in some cases (23,28-30). It is valid to compare the different Affibody molecules since the backbones of the three Affibody molecules are identical. The only differences are alternative amino acids at the randomized positions in the antigen-binding region.

The applied methods in this study have been well described previously. Biacore analysis was used for real-time biospecific interaction studies between the Affibody molecules and HER2 (27). This allowed for a reproducible and reliable determination of the affinities. The radiolabeling of the molecules seemed to not disturb the binding to any significant degree, as indicated by the competitive administration of non-labeled substances to the cultured cells. Immunohistochemical stainings for HER2 were performed as described in several of our previous reports on clinical tumor samples $(36,37)$. The digital image analyses of the autoradiographs were performed using modified versions of previously described procedures and software $(12,38)$.
Cellular binding was analyzed in tumor spheroids composed of breast cancer BT474 cells, which highly express HER2. These cells can be grown as both monolayer cultures and cell spheroids and are an interesting in vitro model of non-vascularized micro-metastases as has previously been reported by Qvarnström et al (12). The spheroid model allows studies of penetration, binding and action of diagnostic and therapy effective substances under strictly controlled conditions. Thus variations in binding of tumor targeting agents due to variations in kidney clearance, vascularization and nutrition supply, host defense and other in vivo factors can be avoided using the spheroid in vitro model $(1,2,8)$. The spheroids used for the experiments in this study had diameters in the range 700-850 $\mu \mathrm{m}$ and at these sizes there was a well-developed proliferation gradient in the outer viable cell layer of the spheroids and a well-developed necrosis in the centre. The HER2 staining was strong in the viable cell layer but had a somewhat lower intensity near the necrotic region. HER2 is overexpressed in several cancers, especially breast cancer and is an interesting target for both diagnostic and therapy purposes (16-18).

The conclusion from the studies in this report is that a binding-site barrier exists in tumor spheroids which can be overcome by increased amounts of substance and by reduced affinity of the substance. The next step is to confirm these results in in vivo models (transplanted tumors and patient biopsies). Results of these studies will indicate a suitable affinity for agents applied for diagnosis and therapy of breast cancer micrometastases, which due to their small size have not vascularized.

\section{Acknowledgements}

This study was financially supported by a grant from the Swedish Cancer Society (contract no. 100255) and a research scholarship from Lennanders Stiftelse, Uppsala, Sweden. The authors thank Veronika Asplund at Imanet AB, Uppsala, Sweden for help with spheroid cultures and autoradiography processing.

\section{References}

1. Fracasso G and Colombatti M:Effect of therapeutic macromolecules in spheroids. Crit Rev Oncol Hematol 36: 159-178, 2000.

2. Carlsson J and Gedda L: Penetration of tumor therapy interesting substances in non-vasularized metastases: review of studies in multicellular spheroids. Curr Cancer Ther Rev 2: 293-304, 2006.

3. Adams GP, Schier R, McCall AM, Simmons HH, Horak EM, Alpaugh RK, Marks JD and Weiner LM: High affinity restricts the localization and tumor penetration of single-chain fv antibody molecules. Cancer Res 61: 4750-4755, 2001.

4. Thurber GM: Kintecis of antibody penetration into tumors. In: Targeted Radionuclide Therapy. Speer TW (ed). Lippincott Williams \& Wilkins, Philadelphia, ISBN 978-0-7817-9693-4. Chapter 12, pp168-181, 2011.

5. Sutherland RM and Durand RE: Radiation response of multicell spheroids - an in vitro tumour model. Curr Top Radiat Res Q 11: 87-139, 1976.

6. Carlsson J and Yuhas JM: Liquid-overlay culture of cellular spheroids. Recent Results Cancer Res 95: 1-23, 1984.

7. Mueller-Klieser W: Multicellular spheroids. A review on cellular aggregates in cancer research. J Cancer Res Clin Oncol 113: 101-122, 1987.

8. Sutherland RM: Cell and environment interactions in tumor microregions: the multicell spheroid model. Science 240: 177-184, 1988. 
9. Mueller-Klieser W: Tumor biology and experimental therapeutics. Crit Rev Oncol Hematol 36: 123-139, 2000.

10. Santini MT, Rainaldi G and Indovina PL: Apoptosis, cell adhesion and the extracellular matrix in the three-dimensional growth of multicellular tumor spheroids. Crit Rev Oncol Hematol 36 : 75-87, 2000

11. Indovina P, Rainaldi G and Santini MT: Three-dimensional cell organization leads to a different type of ionizing radiationinduced cell death: MG-63 monolayer cells undergo mitotic catastrophe while spheroids die of apoptosis. Int J Oncol 31: 1473-1483, 2007

12. Qvarnström OF, Simonsson M, Eriksson V, Turesson I and Carlsson J: GammaH2AX and cleaved PARP-1 as apoptotic markers in irradiated breast cancer BT474 cellular spheroids. Int J Oncol 35: 41-47, 2009.

13. Carlsson $\mathbf{J}$ and Acker $\mathrm{H}$ : Relations between $\mathrm{pH}$, oxygen partial pressure and growth in cultured cell spheroids. Int J Cancer 42: 715-720, 1988

14. Weinstein JN and van Osdol W: Early intervention in cancer using monoclonal antibodies and other biological ligands: micropharmacology and the 'binding site barrier'. Cancer Res 52: S2747-S2751, 1992.

15. Rudnick SI and Adams GP: Affinity and avidity in antibodybased tumor targeting. Cancer Biother Radiopharm 24: 155-161, 2009.

16. Abramson V and Arteaga CL: New strategies in HER2-overexpressing breast cancere: Many combinations of targeted drugs available. Clin Cancer Res, Jan 19, 2011 [Epub ahead of print].

17. Capala J and Bouchelouche K: Molecular imaging of HER2positive breast cancer: a step toward an individualized 'image and treat' strategy. Curr Opin Oncol 22: 559-566, 2010.

18. Tolmachev V: Imaging of HER-2 overexpression in tumors for guiding therapy. Curr Phar Des 14: 2999-3019, 2008.

19. Wikman M, Steffen AC, Gunneriusson E, Tolmachev V, Adams GP, Carlsson J and Ståhl SA: Selection and characterization of HER2/ neu binding affibody ligands. Protein Eng Des Sel 17: 455-462, 2004.

20. Grönwall C, Sjöberg A, Ramström M, Höidén-Guthenberg I, Hober S, Jonasson P and Ståhl S: Affibody-mediated transferrin depletion for proteomics applications. Biotechnol J 2: 1389-1398, 2007.

21. Grönwall C, Jonsson A, Lindström S, Gunneriusson E, Ståhl S and Herne N: Selection and characterization of Affibody ligands binding to Alzheimer amyloid beta peptides. J Biotechnol 128 : $162-183,2007$

22. Friedman M, Nordberg E, Höidén-Guthenberg I, Brismar H, Adams GP, Nilsson FY, Carlsson J and Stahl S: Phage display selection of Affibody molecules with specific binding to the extracellular domain of the epidermal growth factor receptor Protein Eng Des Sel 20: 189-199, 2007.

23. Nord K, Nord O, Uhlén M, Kelley B, Ljungqvist C and Nygren PÅ: Recombinant human factor VIII-specific affinity ligands selected from phage-displayed combinatorial libraries of protein A. Eur J Biochem 268: 4269-4277, 2001.

24. Grönwall C, Snelders E, Palm AJ, Eriksson F, Herne N and Ståhl S: Generation of Affibody ligands binding interleukin-2 receptor alpha/CD25. Biotechnol Appl Biochem 50: 97-112, 2008
25. Wikman M, Rowcliffe E, Friedman M, Henning P, Lindholm L, Olofsson S and Ståhl S: Selection and characterization of an HIV-1 gp120-binding affibody ligand. Biotechnol Appl Biochem 45: 93-105, 2006

26. Sandström K, Xu Z, Forsberg G and Nygren PÅ: Inhibition of the CD28-CD80 co-stimulation signal by a CD28-binding affibody ligand developed by combinatorial protein engineering. Protein Eng 16: 691-697, 2003.

27. Löfblom J, Feldwisch J, Tolmachev V, Carlsson J, Ståhl S and Frejd FY: Affibody molecules: engineered proteins for therapeutic, diagnostic and biotechnological applications. FEBS Lett 584: 2670-2680, 2010

28. Friedman M, Orlova A, Johansson E, Eriksson TL, HöidenGuthenberg I, Tomachev V, Nilsson FY and Ståhl S: Directed evolution to low nanomolar affinity of a tumor-targeting epidermal growth factor receptor-binding affibody molecule. J Mol Biol 376: 1388-1402, 2008.

29. Gunneriusson E, Nord K, Uhlén M and Nygren PÅ: Affinity maturation of a Taq DNA polymerase specific affibody by helix shuffling. Protein Eng 12: 873-878, 1999.

30. Orlova A, Magnusson M, Eriksson TL, Nilsson M, Larsson B, Höiden-Guthenberg I, Widström C, Carlsson J, Tolmachev V, Ståhl S and Nilsson FY: Tumor imaging using a picomolar affinity HER 2 binding affibody molecule. Cancer Res 66: 4339-4348, 2006.

31. Nilsson FY and Tolmachev V: Affibody molecules: new protein domains for molecular imaging and targeted tumor therapy. Curr Opin Drug Discov Devel 10: 167-175, 2007.

32. Tolmachev V, Orlova A, Nilsson FY, Feldwisch J, Wennborg A and Abrahmsen L: Affibody molecules: potential for in vivo imaging of molecular targets for cancer therapy. Expert Opin Biol Ther 7: 555-568, 2007.

33. Orlova A, Feldwisch J, Abrahmsen L and Tolmachev V: Update: affibody molecules for molecular imaging and therapy for cancer. Cancer Biother Radiopharm 22: 573-584, 2007.

34. Tran TA, Rosik D, Abrahmsen L, Sandström M, Sjöberg A, Wållberg H, Ahlgren S, Orlova A and Tolmachev V: Design, synthesis and biological evaluation of a multifunctional HER2specific Affibody molecule for molecular imaging. Eur J Nucl Med Mol Imaging 36: 1864-1873, 2009.

35. Nordberg E, Friedman M, Göstring L, Adams GP, Brismar H, Nilsson FY, Ståhl S, Glimelius B and Carlsson J: Cellular studies of binding, internalization and retention of a radiolabeled EGFRbinding affibody molecule. Nucl Med Biol 34: 609-618, 2007.

36. Wei Q, Sheng L, Shui Y, Hu Q, Nordgren $\mathrm{H}$ and Carlsson J: EGFR, HER2 and HER 3 expression in laryngeal primary tumours and corresponding metastases. Ann Surg Oncol 15: 1193-1201, 2008.

37. Wei Q, Shui Y, Zheng S, Wester K, Nordgren H, Nygren P, Glimelius B and Carlsson J: EGFR, HER2 and HER3 expression in primary colorectal carcinomas and corresponding metastases. Implications for targeted radionuclide therapy. Oncol Rep 25: 3-11, 2011.

38. Qvarnström OF, Simonsson M, Johansson KA, Nyman J and Turesson I: DNA double strand break quantification in skin biopsies. Radiother Oncol 72: 311-317, 2004. 